\title{
Sound power determination for centrifugal pumps used for local agricultural irrigation in Romania
}

\author{
Mihai Chițoiu ${ }^{1}$, Georgiana Moiceanu ${ }^{2 *}$, Gigel Paraschiv ${ }^{1}$, Mădălina Ștefan $^{1}$ and Andreea \\ Matache $^{3}$ \\ ${ }^{1}$ University POLITEHNICA from Bucharest, The Faculty of Biotechnical Systems Engineering, 313 \\ Splaiul Independentei, Bucharest, 060042, Bucharest, Romania \\ ${ }^{2}$ University POLITEHNICA from Bucharest, Faculty of Entrepreneurship, Business Engineering and \\ Management, 313 Splaiul Independentei, Bucharest, 060042, Bucharest, Romania \\ ${ }^{3}$ National Research - Development Institute for Machines and Installations Designed to Agriculture \\ and Food Industry, 6 Ion Ionescu de la Brad Blv., 013813 Bucharest, Sector 1, Romania
}

\begin{abstract}
Technological enhancements in the domain of agriculture have led to easing the burden of physical work and increased productivity. Irrigation has always been one of the most crucial elements needed for a high yielding crop, given the current uncertainty of climate change, where climate is increasing in unpredictability. Even though a wide array of methods has been developed in recent years, for the Romanian local agricultural irrigation, a popular method remains using motor-pumps. But the issue with this type of equipment is that during active functioning, it produces high sustained levels of noise pollution, and the risk of operator hearing damage is relatively unknown for long term exposures (years). In this paper, the levels of acoustic power of an irrigation motor-pump model WHT-60 were determined, taking into consideration the required standards, and directives, necessary for this activity, imposed by the European Union for all member countries.
\end{abstract}

\section{Introduction}

Technological advances in the domain of agriculture have increased productivity rates and have lowered physical workload for operators. Also, these technological advances, have lowered the number of personnel needed for a successful business, through acquisitions of new and advanced equipment types for specific activities. Farming is at the dawn of a major transformation not only in technology and production practices, but also in size of business, resource control and operation [1]. Constant exposure to the noise levels of these new implemented agricultural machines can cause hearing problems for operators. Exposure to noise from agricultural tools and machinery can cause partial, or even permanent hearing loss, and tinnitus. [2] Hearing loss can lead to a depreciation of life quality, and some early signs of hearing loss can include ringing or buzzing noise in the ears after work, difficulties in understanding a conversation, failing to hear background noise etc.

*Corresponding author: georgiana.moiceanu@upb.ro 
Sound is a radiant energy that is transmitted through space by longitudinal pressure and it is the objective cause of hearing, and the measurement of sound is related to pressure, frequency and duration, commonly measured in decibel units [3]. Generally, the effects of noise are hidden between $30-65 \mathrm{~dB}$, but exposure to sounds of over $85 \mathrm{~dB}$ can cause physical long-term effects, like an increase in blood pressure, decrease of heart pulse, weak muscles and retraction of blood from the skin [4].

Noise pollution in agriculture is not a new issue, its threat being recognized by the National Occupational Safety and Health, which conducted a National Occupational Exposure Survey, between 1981 and 1983, to provide descriptive data of occupational safety and health conditions, leading to the statement that workers were considered exposed to noise pollution if the noise levels were $85 \mathrm{~dB}$ or higher, regardless of exposure duration [5]. In a recent study, most farmers reported exposure to high noise levels for more than eight hours during agricultural activities, therefore noise is a real potential danger for the quality of life [6].

Previous studies have shown that greater risks for hearing impairment come from using medium to low-power tractors $(84-101 \mathrm{~dB})$, which unfortunately are frequently used in private farms, as well as combine harvesters (88-92 dB) [7]. Agricultural equipment manufacturers are constantly developing ways to reduce sound levels reached in the vicinity of operators [8].

In one study, in which 31 agricultural equipment types were tested for noise pollution at operator's ear level, the results showed the need for continued application of noise reduction techniques, in order to protect the hearing of the operator [9]. Researchers, following their researches, seem to come to a similar conclusion, in which hearing discomfort depends on magnitude, frequency, direction and duration of the exposed noise to the ear of the operator [10]

In Romania, monitoring of noise pollution is under the attributions of the Ministry of Environment, which outlined a rise in the average noise levels, from $50 \mathrm{~dB}$, at the beginning of the 80 's to $70 \mathrm{~dB}$, in the year 1999. Romania, being part of the European Union, is applying European Directives and Standards with the sole purpose to limit the rise of noise levels, to protect the operator hearing, and to bring in new equipment that is tested and certified as within legal limits of noise emissions by equipment type [11]. When it comes to agricultural equipment, tests are realized following the instructions of Directive 2000/14/EC [12] and, of Standard SR EN ISO 3744/2011, for determining the levels of acoustic power and acoustic energy of noise sources using acoustic pressure [13]. There are studies performed using different measurement surfaces according to previously mentioned noise measurement regulations, in which different agricultural machinery were tested. In one study, the authors investigated the noise level of a motor hoe, basically having the same noise source as in our study, identified as the thermal engine, using the same testing methodology provided in Directive 2000/14/EC which will be used in our study [14]. However, the measuring surface was of hemispherical type fact which had an impact on the microphones positioning during measurements. Another study about the noise produced by a brush cutter took into consideration various working conditions, the measurement surface being also considered as hemispherical [15]. The obtained results highlighted the need for workers protection measures against the high noise produced by the equipment.

The aim of this study was to measure the acoustic power level of an irrigation motorpump in order to establish the risks at which its operator is subjected during functioning. There were made several measurements with the water pump unit set for three output pumping heights: 10, 20 and respectively 30 meters. The measurement surface was chosen to be on a parallelepiped surface according to the noise test code imposed for this water pump unit. 


\section{Materials and Method}

Tests were realized within the testing laboratory of National Institute for Research and Development of Agricultural Machines.

Also, given Romania's status as a member of European Union, for testing purposes we followed Directive 2000/14/EC and Standard SR EN ISO 3744/2011.

During tests we measured the noise levels of an irrigation equipment type used in Romania, a centrifugal pump type WTH 60 equipped with an internal combustion engine. These pumps are popular in Romania and are used for irrigation purposes, as well as for eliminating water from floods. It is driven by an OHV petrol motor, type $168 \mathrm{~F}-1$, air cooled, with a gas reservoir capacity of 3.6 litres.

Centrifugal pump WHT 60 is composed out of a petrol engine and a water pump - the pump is fixed on a frame with a shock absorbent device. The pump itself is composed out of pump body, pump lid, flow paths and the rotor. Pump aspiration is realized with a onesided valve which does not allow liquid to penetrate the body of the pump.

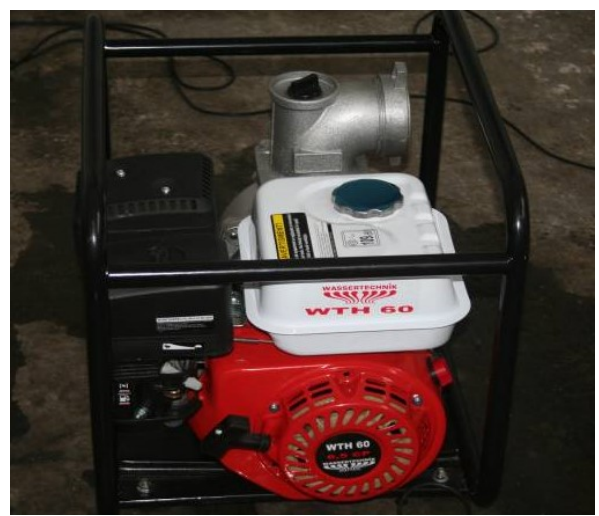

Fig. 1. Centrifugal pump equipped with an internal combustion engine WHT 60.

Table 1. Main Characteristics of the centrifugal pump WHT 60

\begin{tabular}{|c|c|c|c|}
\hline No. & Characteristics & Measuring Units & Value \\
\hline 1 & Motor type & - & OHV, petrol \\
\hline 2 & Net weight & $\mathbf{k g}$ & $\mathbf{3 0}$ \\
\hline 3 & Maximum pumping height & $\mathbf{m}$ & $\mathbf{3 0}$ \\
\hline 4 & Maximum flow & $\mathbf{m} / \mathbf{h}$ & $\mathbf{6 0}$ \\
\hline 5 & Cylinder capacity & $\mathbf{c c}$ & $\mathbf{1 9 6}$ \\
\hline 6 & Compression relation & $\mathrm{HP} / \mathbf{r p m}$ & $\mathbf{8 . 5 : 1}$ \\
\hline 7 & Maximum power & $\mathrm{HP} / \mathbf{r p m}$ & $\mathbf{6 . 5 / 3 6 0 0}$ \\
\hline 8 & Reservoir capacity & $\mathrm{I}$ & $\mathbf{3 . 6}$ \\
\hline
\end{tabular}

\subsection{Testing conditions}

In order to assure a high degree of certitude for the results, the following operations were realized on the centrifugal pump WHT 60:

- the lapping of the equipment was realized according to its instructions manual;

- detachable and undetachable parts were verified to ensure the relevance of the tests;

- oil levels and petrol levels were checked;

- the motor was run for 30 minutes before performing measurements. 


\subsection{Equipment and measuring devices used for testing}

In order to accurately measure the noise levels of the WHT 60 pump, the following devices were used:

Table 2. Equipment types and devices being used for testing

\begin{tabular}{|c|c|c|}
\hline No. & Name of the instrument of device & Measuring domain \\
\hline 1 & Roulette & $0 \div 8 \mathrm{~m}$ \\
\hline 2 & PULSE System with microphones & $100 \div 10000 \mathrm{Hzkg}$ \\
\hline 3 & Thermo-hygrometer DH 50 & $\begin{array}{c}\text { Humidity: } 5 \div 95 \% \\
\text { Temperature: } 20 \div 80^{\circ} \mathrm{C}\end{array}$ \\
\hline 4 & Anemometer Testovent 4000 & $0,4-40 \mathrm{~m} / \mathrm{s}$ \\
\hline
\end{tabular}

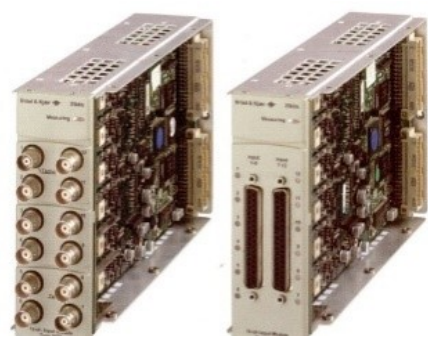

Fig. 2 PULSE Data acquisition board - 12 channels.

\subsection{Pulse System microphone distribution}

Taking into consideration the instructions from Directive 2000/14/EC and standard SR EN $3744 / 2011$, the microphone distribution method was chosen to be on a parallelepiped surface with measurement distance $\mathrm{d}=1 \mathrm{~m}$ from the reference box. The latter was a hypothetical surface defined by the smallest right parallelepiped that enclosed the noise source under test, namely the pump. In figure 3 , is presented the reference box on one reflecting plane where $l_{1}, l_{2}, l_{3}$ are the length, width and height of the reference box and $d_{0}$ represents the characteristic source dimension.

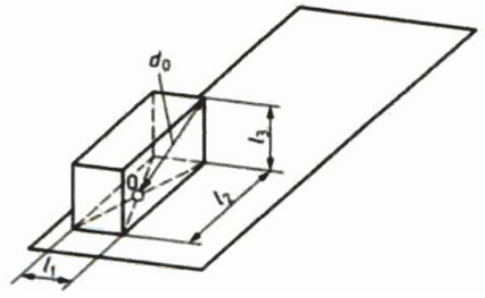

Fig. 3. Reference box on one reflecting plane [13].

The area $\mathrm{S}$ of the measurement surface was computed using relation (1).

where

$$
S=4(a b+b c+c a)
$$

$a=0.5 l_{1}+d$

$b=0.5 l_{2}+d$

$c=l_{3}+d$ 


\subsection{Determining the acoustic power level of the WHT 60 centrifugal pump}

The time-averaged sound pressure level $L_{p i(S T)}$ for the pump noise source under test was measured for each $i$-th microphone using the A scale for weighting. The measurement time interval was set at 15 seconds. There were made 3 replicas of the measurements. During the same test the time-averaged sound pressure level of the background noise, $L_{p i(B)}$ was measured before and after the 3 noise test replicas in the same conditions but with the noise source shut down. The mean time-averaged sound pressure level $L_{P(S T)}$ in decibel was then computed using relation (2).

$$
L_{P(S T)}=10 \lg \left[\frac{1}{N_{m}} \sum_{i=1}^{N_{m}} 10^{0.1 L_{p i(S T)}}\right]
$$

where $N_{m}$ is the number of microphones used for the measurement.

The mean time-averaged sound pressure level of the background noise, $L_{P(B)}$ was calculated using relation (3).

$$
L_{P(B)}=10 \lg \left[\frac{1}{N_{m}} \sum_{i=1}^{N_{m}} 10^{0.1 L_{p i(B)}}\right]
$$

The corrections for the background noise, $K_{l}$ was computed using relation (4):

$$
K_{1}=-10 \lg \left(1-10^{-0.1 \Delta L_{P}}\right)
$$

where $\Delta L_{P}=L_{P(S T)}-L_{P(B)}$.

The background noise correction condition was that if $\Delta L_{P}>15 d B$, then $K_{l}$ will be assumed to be zero, if $6 d B \leq \Delta L_{P} \leq 15 d B$ then the corrections will be made according to relation (4) and in all the other cases the tests will be unvalidated and renewed.

Afterward the surface time-averaged sound pressure level $L_{P}(\mathrm{~dB})$ was computed using relation (5):

$$
L_{P}=L_{P(S T)}-K_{1}
$$

The sound power level for the WHT 60 centrifugal pump was determined using relation (6):

where $S_{0}=1 \mathrm{~m}^{2}$.

$$
L_{W}=L_{P}+10 \lg \frac{s}{S_{0}}
$$

Sound power level was determined using the PULSE system, equipped with 9 microphones and distributed according to the instructions from SR EN ISO 3744/2011.

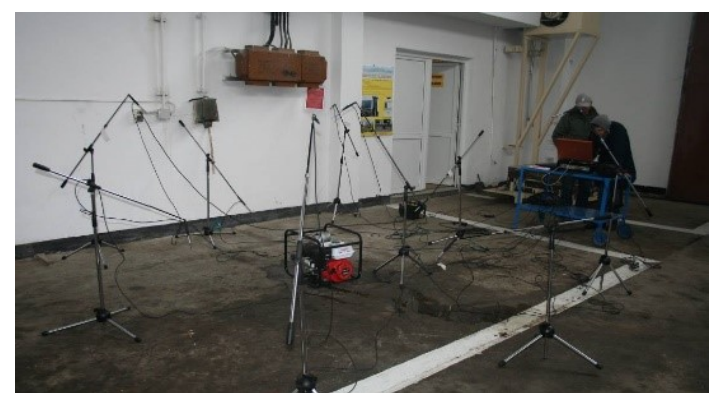

Fig. 4. Microphone distribution for determining the acoustic power of the PULSE system (9 microphones).

\section{Results and discussion}

The measuring surface $S$ was calculated based on the dimensions of the noise source, and the value of $19.81 \mathrm{~m}^{2}$ was obtained. Following the microphone distribution around the 
centrifugal pump WHT 60, according to the instructions of SR EN 3744/2011, the equipment was turned on and the data acquisition was performed.

There were performed three sets of measurements on the water pump at three pumping heights, 10, 20 and respectively maximum 30 meters, in order to asses the acoustic power level in function of the load sustained by the unit.

The acoustic pressure and acoustic power results obtained for the $10 \mathrm{~m}$ pumping height, are presented in table 3 .

Table 3. Experimental data for sound power determination for $10 \mathrm{~m}$ pumping height

\begin{tabular}{|l|l|l|l|l|l|l|l|l|l|l|l|}
\hline & $\begin{array}{l}L_{p l(S T)} \\
(d B)\end{array}$ & $\begin{array}{l}L_{p 2(S T)} \\
(d B)\end{array}$ & $\begin{array}{l}L_{p 3(S T)} \\
(d B)\end{array}$ & $\begin{array}{l}L_{p 4(S T)} \\
(d B)\end{array}$ & $\begin{array}{l}L_{p 5(S T)} \\
(d B)\end{array}$ & $\begin{array}{l}L_{p 6(S T)} \\
(d B)\end{array}$ & $\begin{array}{l}L_{p 7(S T)} \\
(d B)\end{array}$ & $\begin{array}{l}L_{p 8(S T)} \\
(d B)\end{array}$ & $\begin{array}{l}L_{p 9(S T)} \\
(d B)\end{array}$ & $\boldsymbol{L}_{\boldsymbol{P}(d \boldsymbol{B})}$ & $\boldsymbol{L}_{\boldsymbol{W}(d B)}$ \\
\hline Background & 38.5 & 38.6 & 39.1 & 39.2 & 39.4 & 39.2 & 39.7 & 39.2 & 38.6 & & \\
\hline Replica 1 & 85.91 & 88.03 & 83.28 & 88.19 & 85.04 & 85.08 & 87.84 & 86.92 & 87.03 & $\mathbf{8 6 . 6 3}$ & $\mathbf{9 9 . 6}$ \\
\hline Replica 2 & 85.48 & 86.43 & 83.85 & 88.63 & 85.12 & 86.34 & 88.09 & 87.80 & 86.54 & $\mathbf{8 6 . 7 0}$ & $\mathbf{9 9 . 7}$ \\
\hline Replica 3 & 85.38 & 86.90 & 83.93 & 88.48 & 85.86 & 86.87 & 89.06 & 86.81 & 88.37 & $\mathbf{8 7 . 1 1}$ & $\mathbf{1 0 0 . 1}$ \\
\hline Background & 37.8 & 37.2 & 37.2 & 36.9 & 37.4 & 36.8 & 36.4 & 36.6 & 37.3 & & \\
\hline
\end{tabular}

In table 4 are presented the results obtained for the $20 \mathrm{~m}$ pumping height.

Table 4. Experimental data for sound power determination for $20 \mathrm{~m}$ pumping height

\begin{tabular}{|l|l|l|l|l|l|l|l|l|l|l|l|}
\hline & $\begin{array}{l}L_{p 1(S T)} \\
(d B)\end{array}$ & $\begin{array}{l}L_{p 2(S T)} \\
(d B)\end{array}$ & $\begin{array}{l}L_{p 3(S T)} \\
(d B)\end{array}$ & $\begin{array}{l}L_{p 4(S T)} \\
(d B)\end{array}$ & $\begin{array}{l}L_{p 5(S T)} \\
(d B)\end{array}$ & $\begin{array}{l}L_{p 6(S T)} \\
(d B)\end{array}$ & $\begin{array}{l}L_{p 7(S T)} \\
(d B)\end{array}$ & $\begin{array}{l}L_{p 8(S T)} \\
(d B)\end{array}$ & $\begin{array}{l}L_{p 9(S T)} \\
(d B)\end{array}$ & $\boldsymbol{L}_{\boldsymbol{P}(d \boldsymbol{B})}$ & $\boldsymbol{L}_{\boldsymbol{W}(\boldsymbol{d} \boldsymbol{B})}$ \\
\hline Background & 38.2 & 38.1 & 38.4 & 38.7 & 39.1 & 39.1 & 39.2 & 38.1 & 38.4 & & \\
\hline Replica 1 & 85.42 & 87.16 & 84.46 & 88.83 & 85.89 & 86.87 & 88.39 & 88.45 & 88.31 & $\mathbf{8 7 . 3 1}$ & $\mathbf{1 0 0 . 3}$ \\
\hline Replica 2 & 85.24 & 87.80 & 84.39 & 89.06 & 85.25 & 86.24 & 88.41 & 88.51 & 88.19 & $\mathbf{8 7 . 3 0}$ & $\mathbf{1 0 0 . 3}$ \\
\hline Replica 3 & 84.80 & 87.25 & 84.99 & 88.17 & 85.56 & 86.01 & 88.69 & 87.87 & 87.87 & $\mathbf{8 7 . 0 1}$ & $\mathbf{1 0 0 . 0}$ \\
\hline Background & 36.2 & 36.8 & 36.5 & 36.1 & 36.7 & 36.5 & 36.9 & 36.9 & 37.2 & & \\
\hline
\end{tabular}

Final results obtained for the maximum pumping height, based on data acquisition of time-averaged sound pressure level $L_{p i(S T)}$ and the results calculated are presented in table 5 .

Table 5. Experimental data for sound power determination for $30 \mathrm{~m}$ pumping height

\begin{tabular}{|l|l|l|l|l|l|l|l|l|l|l|l|}
\hline & $\begin{array}{l}L_{p l(S T)} \\
(d B)\end{array}$ & $\begin{array}{l}L_{p 2(S T)} \\
(d B)\end{array}$ & $\begin{array}{l}L_{p 3(S T)} \\
(d B)\end{array}$ & $\begin{array}{l}L_{p 4(S T)} \\
(d B)\end{array}$ & $\begin{array}{l}L_{p 5(S T)}(d B) \\
(d B)\end{array}$ & $\begin{array}{l}L_{p 6(S T)} \\
(d B)\end{array}$ & $\begin{array}{l}L_{p 7(S T)} \\
(d B)\end{array}$ & $\begin{array}{l}L_{p 8(S T)} \\
(d B)\end{array}$ & $\begin{array}{l}L_{p 9(S T)} \\
(d B)\end{array}$ & $\boldsymbol{L}_{\boldsymbol{P}(d \boldsymbol{B})}$ & $\boldsymbol{L}_{\boldsymbol{W}(d \boldsymbol{B})}$ \\
\hline Background & 39.5 & 38.4 & 39.4 & 39 & 39.9 & 39.2 & 39.8 & 39.1 & 39.6 & & \\
\hline Replica 1 & 86.9 & 89.1 & 85.7 & 90.5 & 86.6 & 87.4 & 89.5 & 89.2 & 89 & $\mathbf{8 8 . 4 6}$ & $\mathbf{1 0 1 . 4}$ \\
\hline Replica 2 & 87.1 & 89.4 & 85.8 & 90.9 & 86.6 & 87.4 & 89.4 & 89.6 & 89.3 & $\mathbf{8 8 . 6 8}$ & $\mathbf{1 0 1 . 6}$ \\
\hline Replica 3 & 87.6 & 89.6 & 86.2 & 91.4 & 87.4 & 88.1 & 89.9 & 90.3 & 90.1 & $\mathbf{8 9 . 2 4}$ & $\mathbf{1 0 2 . 2}$ \\
\hline Background & 35.8 & 35.8 & 36.2 & 36.5 & 38.1 & 36 & 36.9 & 36.6 & 37.1 & & \\
\hline
\end{tabular}

Analysing the obtained results for the three working conditions we conclude that the acoustic power level was maximum for the maximum pumping height. This is explained by the fact that the thermal engine auto-controller was increasing the throttle as the load was increased, thus increasing the noise level. However, the noise level observed in all three working conditions was still high, exceeding $87 \mathrm{~dB}$ sound pressure level and $99 \mathrm{~dB}$ for the acoustic power. Sounds above $85 \mathrm{~dB}$ are harmful for the human ear depending on the period of exposure and occurrence. The workers should wear hearing protection, such as earplugs or earmuffs.

The mean value of the sound power for the maximum pumping height $L_{W(d B)}$ was computed and the result of $101.9 \mathrm{~dB}$ was rounded at $102 \mathrm{~dB}$ weighted on the A scale. 


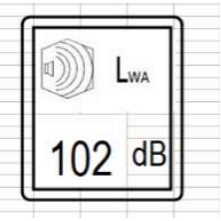

Fig.5 Sound power obtained for WHT 60 centrifugal pump.

\section{Conclusions}

Following the results obtained from determining acoustic power levels of a running WHT 60 centrifugal pump the following conclusions were drawn:

- Even though, according to the Directive 2000/14/EC, this device is emitting an acoustic power level within the legal limits, $102 \mathrm{~dB}$ is still a very high number and the worker acoustic protection is required.

- Increasing the load on the water unit pump has as result also an increase of the noise level, fact which should be considered when operating the pump.

- Long-time exposure and working in the close vicinity of this equipment type, can have negative effects on the operator's hearing health, in the long term.

- Current EU directives and standards take into consideration short to medium time exposure to noise levels generated by different equipment types.

- For effects of noise levels in regard to long time constant exposure, a multitude of data gatherings must be achieved, over a long period of time, in order to better understand these effects on the human hearing health.

- Using this equipment type for irrigation purposes during a drought would subject the operator to a higher incidence of being exposed to these high noise levels, and his hearing health could decrease;

- Further tests, on the topic of operator continuous noise level exposure must be realized, in order to raise awareness on the topic of hearing health risks, better understand how hearing is affected, and impose clearer and stricter rules.

\section{ACKNOWLEDGEMENT}

This work has been funded by the European Social Fund from the Sectoral Operational Programme Human Capital 2014-2020, through the Financial Agreement with the title "Scholarships for entrepreneurial education among doctoral students and postdoctoral researchers (Be Antreprenor!)", Contract no.51680/09.07.2019 - SMIS code: 124539.

\section{References}

[1] A. Gray, M.D. Boehlje, Department of Agricultural Economics, Working Paper 07 (2007)

[2] Government of Western Australia, Noise in agriculture - Identification, assessment and control (2004)

[3]. M.R. Dorgut, H Celen, Noise Levens of Various Agricultural Machineries, Pakistan Journal of Biological Sciences 7, pp. 895-901 (2004;)

[4] A. Sabanci, E. Uz, Ergonomy ve Agricultural Mechanization, $1^{\text {st }}$ International Symposium of Ergonomy (1984)

[5] D. Pessina, M. Guerretti, Effectiveness of Hearing Protection Devices in the Hazard Reduction of Noise from Used Tractors, J. Agric. Eng. Res., pp. 73-80 (2000) 
[6] D. Karamousantas, T Varzakas, A. Kanakis, Noise Levels Produced by Agricultural Machineryand Different Farming Processes, International Journal of Acoustics and Vibrations (2009)

[7] L. Solecki, Duration of exposure to noise among farmers as an important factor of occupational risk, Ann. Agric. Environ. Med., pp 89-93 (2000)

[8] C.W. Suggs, Noise Characteristics of Field Equipment, ASAE (1987)

[9] S.K. Broske, D.A. Hansen, R.L. Strand, D.T. Stueland, Hearing Loss Among High School Farm Students, Am. J. Pub. Health, pp. 619-622 (1989)

[10] A.K. Junoh, Z.M. Nopiah, W. Muhamed, A Study on the Effects of Tyre Vibration to the Noise in Passenger Car Cabin. AMO - Advanced Modelling and Optimization, 13, pp. 53-69 (2011)

[11] CECE (COMMITTEE FOR EUROPEAN CONSTRUCTION EQUIPMENT) Position on the future of the noise emission in the environment by equipment for use outdoors (Directive 2000/14/EC), Brussels, 18 June 2009

[12] Directive 2000/14/EC of the European Parliament and of the Council of 8 May 2000 on the approximation of the laws of the Member States relating to the noise emission in the environment by equipment for use outdoors, Official Journal L 162, 03/07/2000 P. 0001 0078 (2000);

[13] Standard SR EN 3744/2011, Determination of sound power levels and sound energy levels of noise sources using sound pressure - Engineering methods for an essentially free field over a reflecting plane (2011)

[14] V. Vladut, D. Manea, S. t. Biri , S. Bungescu, A. Atanasov, Test of an equipment which works in open air according to the requirements of D2000/14/EC, PROCEEDINGS OF THE 35 INTERNATIONAL SYMPOSIUM ON AGRICULTURAL ENGINEERING "Actual Tasks on Agricultural Engineering", pp 405-414, Opatija - Croaţia, (2007)

[15] E. Sorică, V. Vlăduţ, P. Cârdei, C. Sorică, C. Brăcăcescu, Comparative analysis of the noise and vibration transmitted to the operator by a brush cutter, Proceedings of the XIV-th International Conference „ACOUSTICS AND VIBRATION OF MECHANICAL STRUCTURES - AVMS-2017”, published by „Springer Proceedings in Physics”, pp. 165$172(2017)$ 